for $\bar{p}$ :

S. J. Lindenbaum et al., Phys. Rev. Letters 7 (1961) 185; G. von Dardel et al., Phys. Rev. Letters 5 (1960) 333; U.Amaldi et al., Conf. Intern. d'A ix-en-Provence (1961) p. 283;

R. Armenteros et al., Phys.Rev. 119 (1960) 2068;

T. Elioff et al., Phys. Rev. 128 (1962) 869.

4) H. Lehmann, Nuclear Phys. 29 (1962) 300.

5) A.Scotti and D.Y.Wong, Phys. Rev. Letters 10 (1963) 142 .

6) Riazuddin and M. J. Moravcsik, Physics Letters 4 (1963) 243 and references given there.
7) J.K.Perring, Nuclear Phys. 30 (1962) 424.

8) J.Tinlot, Proc. 1960 Int. Conf. on High Energy Physics at Rochester (1960), p. 111.

9) W. M.Preston, R. Wilson and J.C.Street, Phys. Rev. 118 (1960) 579.

10) V.S. Barashenkov, Fortschritte Physik 10 (1962) 205.

11) H.A. Bethe, Ann. Phys. 3 (1958) 190.

12) H.Eggstain and G. Kellner, Proc.Siena Int. Conf. on Elementary Particles (1963);

L, F. Kirillova, V.A.Nikitin, A. Nomofilov, V.A. Sviridov, L.N. Strunov and M. G. Shafranova, Proc. Siena Int. Conf. on Elementary Particles (1963).

\title{
A STATISTICAL INTERPRETATION OF LARGE ANGLE PROTON-PROTON ELASTIC SCATTERING
}

\author{
L. W. JONES
}

The University of Michigan, Ann Arbor, Michigan

Received 10 January 1964

Recent data on the large-angle elastic p-p scattering by Cocconi et al. 1) indicate a small but detectable scattering cross section outside the diffraction peak. This may be interpreted as scattering from a hard core or perhaps in other ways. However we wish to point out that the observed data may also be in agreement with the predictions of a statistical model 2,3$)$. It is possible to make an explicit prediction of the probability that a statistical state, once formed, will decay into its initial state (in this case, two protons). The probability of such a decay is found empirically, on the basis of numerical phase space calculations, to fit an exponentially decaying function of the centre-of-mass energy. It remains to postulate the probability of formation of a compound state, or "compound nucleon" (corresponding to the probability of a "time-like" intermediate state as opposed to a "space-like" intermediate state or peripheral process). This formation probability was estimated in ref. 2) from comparison of observed average multiplicities and observed antinucleon production with the statistical prediction. In ref. 3) the experimental data for largeangle pion nucleon scattering is also compared with the statistical model *. From these sources, a formation probability equal to $1 / E_{\text {c.m. }}$ (in $\mathrm{GeV}$ ) appears to be a reasonable choice. Therefore the overall prediction of the statistical model is that the ratio of "compound-elastic" scattering to the total cross section is given by

* The data included in ref. 3) are superceded by final experimental results 4 ) corresponding to lower cross sections.

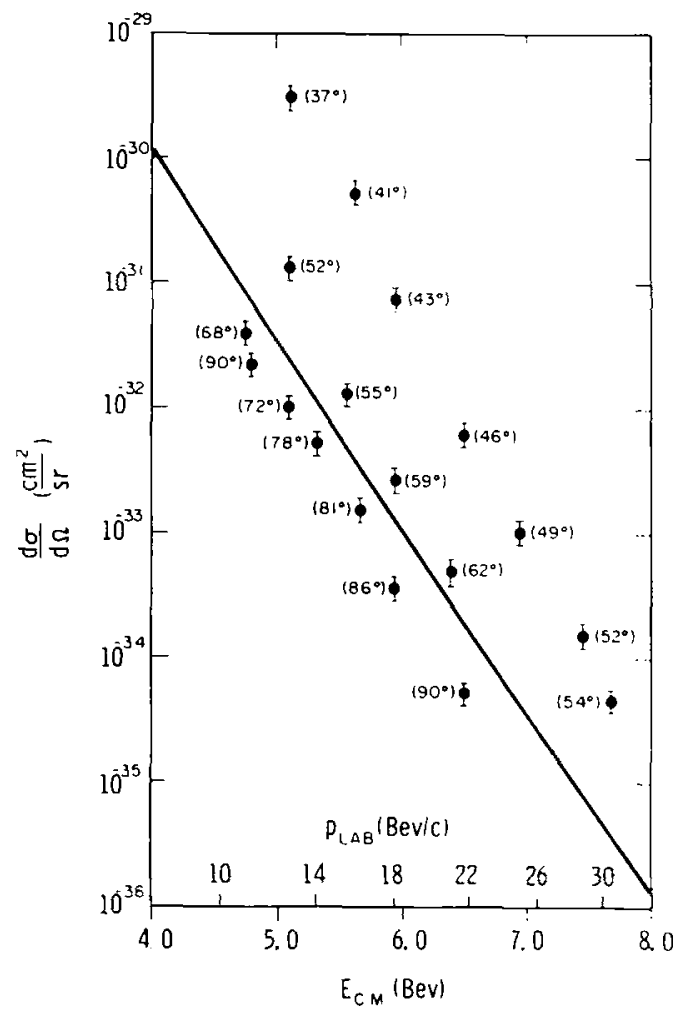

Fig. 1. The data of ref. 1), d $\sigma / d \Omega\left(\mathrm{cm}^{2} / \mathrm{sr}\right)$ plotted versus $E_{\mathrm{c} . \mathrm{m} .}$, together with the prediction of the statistical model (solid curve) given by $\mathrm{d} \sigma / \mathrm{d} \Omega=$

$\left(3 \times 10^{-26 / 2 \pi}\right) E_{\text {c.m. }}{ }^{-1} \exp \left[-3.27\left(E_{\text {c.m. }}-1.88\right)\right]$ The figures in parentheses beside the data points are the corresponding c.m. scattering angles. 


$$
\left\langle\sigma_{E}\right\rangle / \sigma_{\mathrm{S}}=E_{\mathrm{c} . \mathrm{m} .}^{-1} \exp \left[-3.27\left(E_{\mathrm{c} . \mathrm{m} .}-1.88\right)\right],
$$

where $\left\langle\sigma_{E}\right\rangle$ and $\sigma_{\mathrm{S}}$ do not include diffraction

elastic scattering. We will take $\sigma_{\mathrm{S}}=3 \times 10^{-26} \mathrm{~cm}^{2}$ independent of energy.

In fig. 1 we plot the quantity $\mathrm{d}\left\langle\sigma_{E}\right\rangle / \mathrm{d} \Omega$ where

$$
\mathrm{d}\left\langle\sigma_{E}\right\rangle / \mathrm{d} \Omega=\left(3 \times 10^{-26} / 2 \pi\right)\left(\left\langle\sigma_{E}\right\rangle / \sigma_{\mathrm{S}}\right) .
$$

We also plot the experimental data from ref. 1) for comparison. It should be recalled that the statistical model does not predict or even address the question of the angular distribution of products. As may be seen from the figure, the agreement with the statistical prediction is surprisingly good, particularly if we consider the large angle points where $\theta_{\text {c.m. }} \gtrsim 50^{\circ}$. Probably a more sophisticated evaluation of the sticking probability and inclusion of an- gular momentum information in the statistical model are necessary in order to make a more significant comparison. Perhaps the strongest statement that $c$ an be made is that the statistical prediction is as satisfactory an explanation as any other alternative at this time.

Nole added in proof: $\mathrm{G}$. Cocconi has independently reached similar conclusions (private communication).

\section{References}

1) G. Cocconi et al., Phys. Rev. Letters 11 (1963) 499.

2) G. Fast and R.Hagedorn, Nuovo Cimento 27 (1963) 208.

3) G. Fast, R. Hagedorn and L.W. Jones, Nuovo Cimento 27 (1963) 856.

4) Perl, Jones and Ting, Phys. Rev. 132 (1963) 1252.

\author{
$\pi^{ \pm}-\mathrm{p}$ AND $\mathrm{p}-\mathrm{p}$ ELASTIC SCATTERING AT $8.5,12.4$ AND $18.4 \mathrm{GeV} / c$ \\ D. O. CALDWELL *, B. ELSNER **, D. HARTING, \\ A. C. HELMHOLZ ***, W. C. MDDELKOOP and B. ZACHAROV \\ CERN, Geneva, Suitzerland \\ P. DALPIAZ, S. FOCARDI, G. GIACOMELLI ${ }^{+}$and L. MONARI \\ Istituto di Fisica, Universilà di Bologna, \\ Istituto Nazionale di Fisica Nucleare, Sez.Di Bologna \\ J.A. BEANEY, R. A. DONALD and P. MASON \\ Nuclear Physics Research Laboratory, \\ The University of Liverpool, Liverpool \\ L. W. JONES ${ }^{\dagger \dagger}$ \\ Harrison M.Randall Laboralory of Physics, \\ The University of Michigan, Ann Arbor, Michigan
}

Received 8 January 1964

An experiment on elastic scattering, using the spark chamber technique, has been carried out at the CERN proton synchrotron. In this paper results are given for $\pi^{-}-p$ and $p-p$ elastic scattering at incident momenta of $8.5,12.4$ and $18.4 \mathrm{GeV} / c$, and for $\pi^{+}-\mathrm{p}$ at 8.5 and $12.4 \mathrm{GeV} / c$. For these three incident momenta, the measurements cover a range

* Physics Department and Laboratory for Nuclear Sciences, Massachusetts Institute of Technology, Cambridge, Massachusetts.

** Now at DESY, 2 Hamburg - Gr. Flottbek 1, Germany.

*** John Guggenheim Fellow, on sabbatical leave from the University of California, Berkeley, California.

+ Also part-time at CERN, Geneva.

$+\dagger$ Supported in part by the U.S. Office of Naval Research, the Ford Foundation, and the Institute of Science and Technology of the University of Michigan. of values for the square of the four-momentum transfer $|t|$ between $0.12-1.15,0.12-2.36$ and $0.18-4.86(\mathrm{GeV} / \mathrm{c})^{2}$. The limits on $|t|$ were determined by the geometry, which covered all angles between $1.25^{\circ}$ and $7.45^{\circ}$, and by the limited range of the recoil proton in the hydrogen target. Only measurements for $|t|$ values smaller than $1.6(\mathrm{GeV} / \mathrm{c})^{2}$ are reported in this paper.

Besides the intrinsic importance of obtaining data on the fundamental $\pi-p$ and $p-p$ processes, the interest in these phenomena was enhanced by the theoretical predictions of Regge pole theory 1 ). In the limit of high energies and small momentum transfers, the Pomeranchuk trajectory (vacuum trajectory) was expected to dominate. The elastic scattering of any two elementary particles was 